\title{
A case of recurrent headache and neurological deficit
}

\author{
M J G Harrison, J L J M Teepen
}

Presented at the Conference of the Association of British Neurologists and Nederlandse Vereniging voor Neurologie, Aviemore, 4-6 May 1994.

\section{Case presentation}

PROFESSOR M J G HARRISON

The case concerns a woman aged 43 who was admitted to the neurological department in May 1988 with a two week history of progressive headache and mild fever. Subsequently she developed pain and weakness of her legs and difficulties with micturition. She also complained of numb spots on her back. On examination, there was slight ataxia of gait with impaired coordination of her arms and legs. Deep tendon reflexes of her arms and legs were increased. Her temperature was $38^{\circ} \mathrm{C}$. She then became confused with nuchal rigidity and a paraparesis. She also developed respiratory failure for which intubation and mechanical ventilation were temporarily required. Because of positive Mycoplasma pneumoniae antibodies in the blood she was treated with antibiotics. Her condition gradually improved and she was discharged after five weeks. There were some residual deficits including slight gait ataxia, increased deep tendon reflexes in the legs, and urinary incontinence necessitating a suprapubic indwelling catheter. The following investigations were abnormal: in blood; erythrocyte sedimentation rate $26 \mathrm{~mm} / \mathrm{h}$, leucocytes $14.7 \times 10^{9} / 1$, rise in titre of Mycoplasma IgG antibodies ( $1 / 8$ to $1 / 64$ in 11 days), IgM antibodies negative. In CSF; 500 white cells $/ \mathrm{mm},{ }^{3} 80 \%$ mononuclear, protein $0.77 \mathrm{~g} / 1$ (normal $<0.50 \mathrm{~g} / 1$ ), lactate concentration $3.6 \mathrm{mmol} / 1$ (normal $<2.0$ $\mathrm{mmol} / \mathrm{l}$ ). Serological tests of blood and CSF for syphilis, Borrelia burgdorferi, HIV, toxoplasmosis, and Epstein-Barr virus were negative. The EEG showed a diffuse disturbance characterised by nonspecific slow wave activity. Other investigations were normal including chest radiographs, brain $\mathrm{CT}$, and psychometry.

The first point that emerges is that we are dealing with a subacute illness with the features of something inflammatory or even infective with headache, fever, and some meningism. The next is that ataxia with no mention of joint position sense loss and subsequent respiratory failure without muscle weakness all point to localisation in the brain stem. There is probably a second lesion in the spinal cord as although the paraparesis recovered, the bladder never did. On the face of it, therefore, the patient has a subacute inflam- matory process affecting the brain stem and cord, with no systemic disease. The last point is important as diseases such as lupus and sarcoid only rarely affect the brain parenchyma without signs of systemic involvement. Also there was no history of ulcers or uveitis to suggest Behçet's disease, which can affect the brain stem.

The investigations disclosed a leucocytosis and a modest rise in erythrocyte sedimentation rate, although perhaps not to the level that would make one think of lupus or an arteritis. The CSF was cellular but the count was probably not high enough for bacterial infection. There was an intriguing increased CSF lactate concentration. Ninety per cent of patients with bacterial meningitis have a CSF lactate over $3.5 \mathrm{mmol} / 1,{ }^{1}$ but the positive predictive value at this level is only about $30 \%$. The Mycoplasma antibodies rose, but not by much, and there was no change in IgM, so I am not convinced about that.

The next important thing that happened is that the patient improved. We do not know if this was spontaneous, as it might have been from a demyelinating or vascular process, or whether it represented a response to the use of antibiotics, as might have been the case with Mycoplasma or Whipple's disease.

The normal CT only helps in so far as it excludes a mass lesion. Cultures for infections that target the brain stem, such as herpes simplex and listeria, were presumably negative, together with blood cultures which should be included whenever listeria is a possibility. There was nothing to suggest cytomegalovirus ventriculitis that can present with brain stem signs, and there were no polymorphs in the CSF which is sometimes a clue to cytomegalovirus infection.

In August 1988 she was readmitted because of progressive headache and neck pain with pain behind the right ear. Again, she had slight pyrexia (around $38^{\circ} \mathrm{C}$ ). Neurological examination disclosed no additional abnormalities. During admission the patient developed a confusional state and became obtunded. Although bacteriological and virological studies were negative, she was treated with antibiotics. She gradually improved and was sent home six weeks later in about the same clinical condition. Additional findings during this stay were: in blood, erythrocyte sedimentation rate $30 \mathrm{~mm} / \mathrm{h}$, leucocytes $13.2 \times 10^{\%} / 1$, lactate concentration 1.7 
$\mathrm{mmol} / \mathrm{l}$ (normal $0 \cdot 7-1 \cdot 8 \mathrm{mmol} / \mathrm{l}$ ), $\mathrm{B} 1, \mathrm{~B} 6$, and $\mathrm{B} 12$ concentrations within normal limits. In CSF; 100 white cells $/ \mathrm{mm},{ }^{3} 90 \%$ mononuclear, increased protein of $116 \mathrm{mg} / \mathrm{dl}$, slightly increased IgG index of 0.96 (normal $0.40-0.83$ ), lactate concentration 2.9 $\mathrm{mmol} / \mathrm{l}$. Serological studies in blood and CSF were normal including Mycoplasma pneumoniae and Borrelia burgdorferi. The EEG was diffusely irregular with paroxysmal slow wave activity.

So the patient re-presented with what looked like a recrudescence of the original inflammatory illness, only this time consciousness is clouded, so we may be dealing with an increase in parenchymatous damage. The repeat investigations showed that the CSF cell count had now fallen out of the infective range, and with it the lactate had fallen. We now have an increased IgG index but in the presence of a raised CSF protein that is not specific for a demyelinating illness such as multiple sclerosis.

The patient improved again on antibiotics. The negative microbiological studies and serology suggest that this may not be a diagnostic therapeutic response, however.

In January 1989 the patient became ill for the third time complaining of pain behind both ears and in the neck. Furthermore, she had a slowly progressive loss of vision of both eyes. She had increasing difficulty with walking and had noted paraesthesiae in both feet and lower legs. On examination fundoscopy showed pallor of both optic discs. She showed increased ataxia and pyramidal signs of arms and legs with extensor plantar responses. During her stay in hospital the vision of her left eye further deteriorated and she developed a left sixth nerve palsy. She was treated with prednisone followed by some improvement in her condition. During this stay, her blood investigations were normal. Serological and viral studies, including Mycoplasma antibodies were normal, as were extensive immunological studies (including CD4/ CD8 ratio). Visual evoked potential studies were comparable with a bilateral optic neuropathy. Brainstem auditory evoked potentials were also abnormal on both sides especially showing a delayed 3-5 interpeak latency. Median nerve somatosensory evoked potentials were normal. Brain CT was again unremarkable; MRI showed moderate dilatation of the ventricles, and numerous, partly confluent, abnormalities of the white matter including the internal capsule and the corticobulbar and corticospinal tracts in the mesencephalon, pons, and medulla oblongata.

This time there was evidence of more insidious deterioration, and for the first time involvement of the visual pathway. She had symmetric visual loss with optic palor so this is due to bilateral optic nerve lesions. In addition, the ataxia and long tract signs increased so the brain stem lesion was increasing in severity and with the sixth nerve probably also in extent. A battery of evoked potentials was done. I might cynically suggest that they often only tell you what you already know. The visual evoked potential was bound to be abnormal with optic palor and visual loss, and the brainstem auditory evoked potential was going to be abnormal in someone with a brain stem illness. The normal upper limb somatosensory evoked potential is of interest, however, as its normality supports the view that the bladder and lower limb signs are probably indicative of cord pathology.

Does the MRI scan solve the diagnostic puzzle? The higher cuts (fig 1) showed hemispheric white matter disease with a diffuse abnormality with superadded more intense lesions (on T2). There was no mass effect. In the posterior fossa (fig 2) there was considerable T2 hyperintensity in the white matter of the cerebellum and brain stem which was

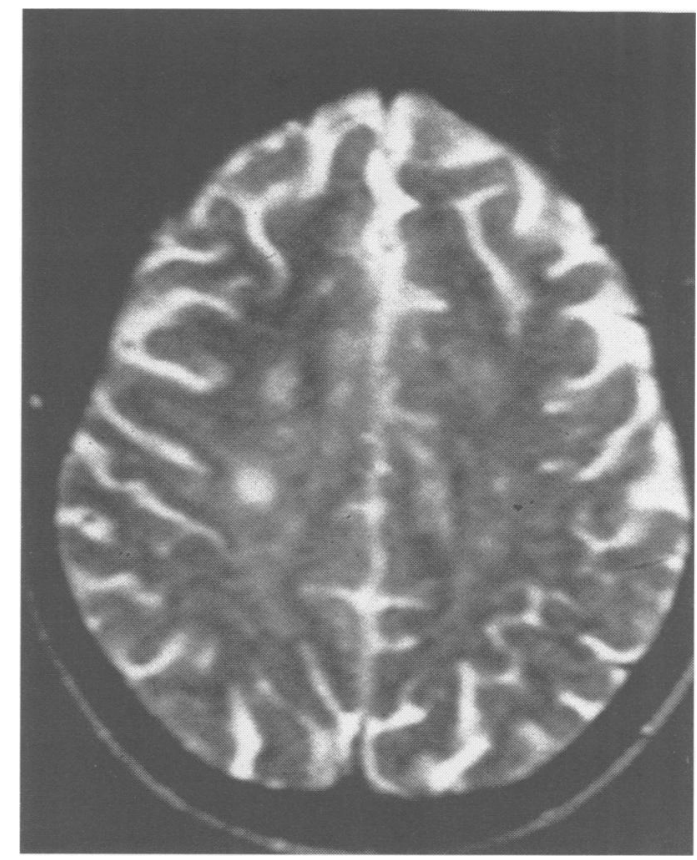

Figure 1 MRI T2 weighted image showing hemispheric white matter lesions.

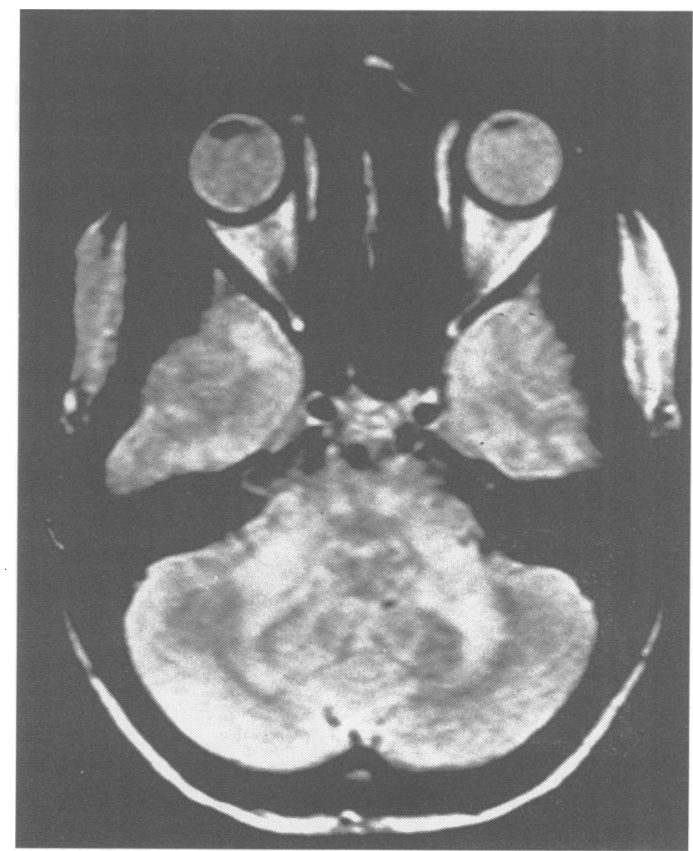

Figure 2 MRI T2 weighted image showing symmetric white matter lesions in the cerebellum and brain stem. 
strikingly symmetric. There were no postgadolinium pictures and that is a pity as meningeal enhancement would have been helpful, and variable enhancement of some but not other white matter lesions would have helped to age some of them, as in multiple sclerosis. Sagittal views would also have been useful in discriminating multiple sclerosis plaques from other white matter lesions. The MRI confirms that we are dealing with a white matter disease with brain stem localisation but does not make a pathological diagnosis.

This time the patient improved on steroids, which is helpful. It suggests that previous improvement was not due to the antibiotics.

Then we have the end stage of the disease.

She stayed in hospital again from April until May 1989 and from June until August 1989. The preexisting symptoms and signs were steadily progressive. At that stage she was almost blind, could only walk with support, and she had increasing headache and pain in the neck by which she was often awakened at night. Apart from severe ataxia, pyramidal features, and impaired abduction of the left eye, she now also had a tremor of both hands. She was treated with prednisone without any improvement. In June she also developed sensory disturbances below the level of T4. Despite treatment with corticosteroids, azathioprine, and vitamins her condition gradually deteriorated and she also developed bilateral hearing loss. The CSF showed 32-68 white cells $/ \mathrm{mm}^{3}$, mainly mononuclear, protein was slightly increased, IgG index normal, lactate concentration $2 \cdot 7 \mathrm{mmol} / \mathrm{l}$. Neither pattern nor light stimulation induced a visual evoked potential on either side.

She was transferred to a rehabilitation centre in August 1989. During the next few months her condition further deteriorated, she became wheelchair bound and almost totally dependent. In January 1990 she was discharged home where she suddenly died in April 1990 during the night.

The brain stem lesion had deteriorated further with increased ataxia, tremor, and hearing loss. The cord lesion had progressed with a level at T4. The visual evoked potentials had gone so the optic nerve lesions had progressed. The CSF was still cellular but there was no persistent IgG increase which is much against multiple sclerosis. The lactate was up a little. The nocturnal death was probably respiratory and due to the brain stem lesion.

\section{Discussion}

This woman had an initially episodic but ultimately progressive illness affecting white matter especially in the brain stem but also affecting the optic nerves and spinal cord. There was an inflammatory component with a cellular CSF. We have had no positive stains or cultures, fever was modest, and there was no early clouding of consciousness, so I doubt if we are dealing with a straightforward meningoencephalitis. We should, however, consider briefly some specific infections. The serology and CD4 counts rule out AIDS. What about Lyme disease? ${ }^{2}$ There was no rash or joint disease, but the early numb patches on her back could have been radicular. Lyme disease normally has a much slower tempo and any CSF pleocytosis is usually less than found here. Also, none of the cells were plasma cells, which can be a clue to Borrelia infection. In any case the antibodies were negative. What about Mycoplasma? I have said why the titres were unimpressive and they were negative through all the later stages of the disease. They were right to pursue Mycoplasma as headache, and a meningoencephalitic illness with brainstem and cord involvement can all occur with one in three victims having residual sequelae, though a relapsing illness would not be expected.

Scattered throughout the case summary are references to an increased lactate. So are we dealing with Leigh's disease, or one of the other mitochondrial cytopathies? They can often affect the brainstem and in fact Leigh's first case had necrotising brain stem lesions. ${ }^{3}$ The optic nerves and cord can be involved, and a respiratory death is not uncommon. Adult cases are sporadic and the lactate can be high in blood, CSF, or both. ${ }^{4}$ High lactate concentrations in acellular CSF samples are particularly suggestive. There are many features that would support such a diagnosis but there are two concerns. A high cell count in the CSF is a little unusual in Leigh's disease and CT and MRI usually show symmetric involvement of the basal ganglia, especially of the putamen.

The commonest cause of episodic then progressive white matter disease in a young female is of course multiple sclerosis, and there are fulminant types such as Marberg's disease. The simultaneous involvement of cord and brainstem in the acute presentation would be unlikely, meningism is rare, simultaneous slow bilateral optic nerve disease unlikely, and there was no persisting increase in CSF IgG. Also the imaging abnormalities were too symmetric.

What about other leukodystrophies ${ }^{5}$ Adult onset adrenoleukodystrophy could explain the distribution of changes but the disease is not usually as aggressive as this, the imaging changes are more diffuse and the CSF is not so cellular. Also she was of the wrong sex!

We must not forget Whipple's disease, which seems to be confined to clinicopathological case conferences in my experience! It tends to affect males, and victims usually but not always have a history of bowel disorder. The leukocytosis and possible response to antibiotics at the first two presentations would be compatible but the localisation is not. Affected patients usually dement with myoclonus and if they have brainstem signs these usually begin with ophthalmoplegia unlike this woman. A one Herz pendular vergence movement of the eyes with synchronous opening and closing of the jaw (oculomasticatory myorhythmia) is said to be pathognomonic of cerebral Whipple's disease. The CSF should be normal although rarely a few cells may be periodic acid Schiff positive.

The inflammatory demyelinating nature of the disease suggests that we could be dealing 
with acute disseminated encephalomyelitis in which meningism and localisation to optic nerve, brainstem, cerebellum, and spinal cord may be seen. ${ }^{6}$ Although acute disseminated encephalomyelitis usually follows exanthems, it can be triggered by upper respiratory tract infections and Mycoplasma. ${ }^{7}$ The recurrences in this illness represent a major argument against the diagnosis, as although recurrences have been described, they seem confined to children.

Can we get any nearer a confident diagnosis from a consideration of the MRI features of the different leukodystrophies? Metachromatic leukodystrophy causes more diffuse hemispheric white matter change then atrophy (and the patient had no peripheral neuropathy). Globoid cell leukodystrophy should have produced high density in the basal ganglia on CT which here was normal. Adrenoleukodystrophy should localise more to the trigone and splenium, and be more homogenous in appearance, and the lesions may enhance. Refsum's disease causes a more diffuse abnormality. In Leigh's disease brainstem localisation is compatible, but the putamen is usually more obviously affected.

The short list contains acute disseminated encephalomyelitis but the poor response to steroids and the recurrences are against that. Could it be Whipple's disease? I think the onset was too acute, the brainstem clinical picture wrong, and the CSF too abnormal. I think that, despite the cellular CSF and lack of imaging abnormality in the basal ganglia, this must be Leigh's disease. The clinical localisation was right, the lactate was increased even when the CSF cell count was modest, and the imaging was appropriately symmetric.

Question

If you had been looking after the patient as well as repeating the MRI with enhancement that you mentioned would you have done anything more invasive, such as a meningeal biopsy?

\section{Response}

Yes, I think I would have done a muscle biopsy looking for ragged red fibres. If that had been normal and the patient continued to deteriorate I would have seriously discussed a brain biopsy.

\section{Question}

What about progressive multifocal leukoencephalopathy?

\section{Response}

The course is more usually relentless although remission may occur in progressive multifocal leukoencephalopathy. The imaging is wrong, though. The lesions of progressive multifocal leukoencephalopathy are of low signal on T1, and trace out the white matter fibre tracts on successive slices. On $\mathrm{T} 2$, the hyperintensity has a characteristic scalloped appearance due to involvement of gyral cores which we did not see here.
DR J L J M TEEPEN

Figure 3 shows the macroscopic features with some discolouration of the basal ganglia and thalamus, which has rather sharp boundaries. Luxol fast blue stained sections of the basal ganglia, pons, and medulla showed discolouration due to demyelination (fig 4). At the level of the spinal cord there was demyelination especially of the corticospinal tract.

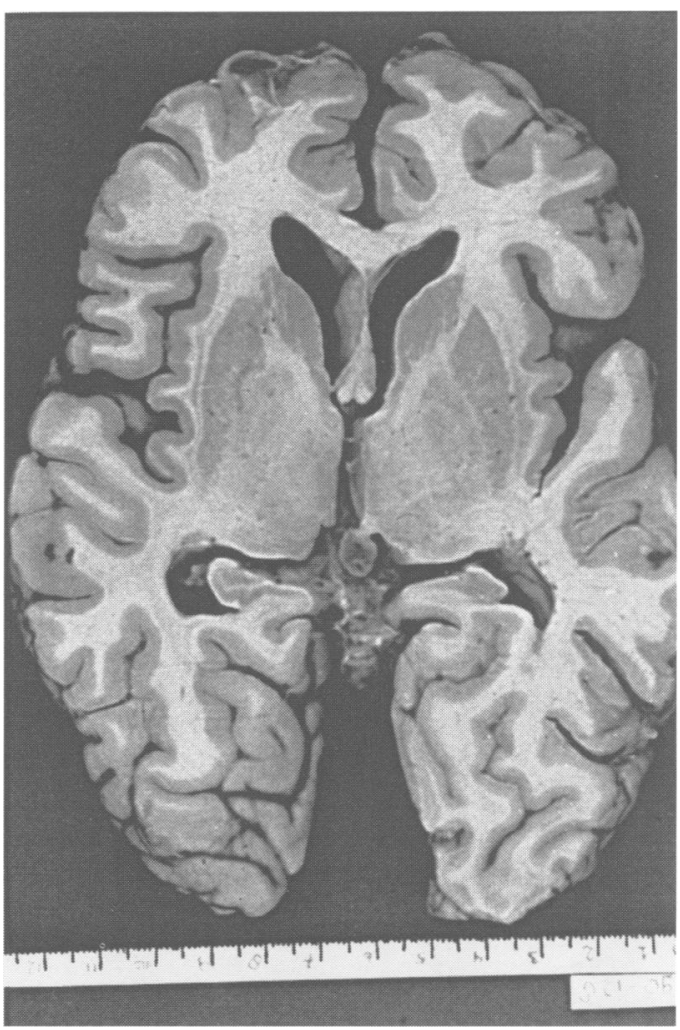

Figure 3 Symmetric demyelination of the globus pallidus and thalamus visible as a grey discolouration on the cut surface of the brain.

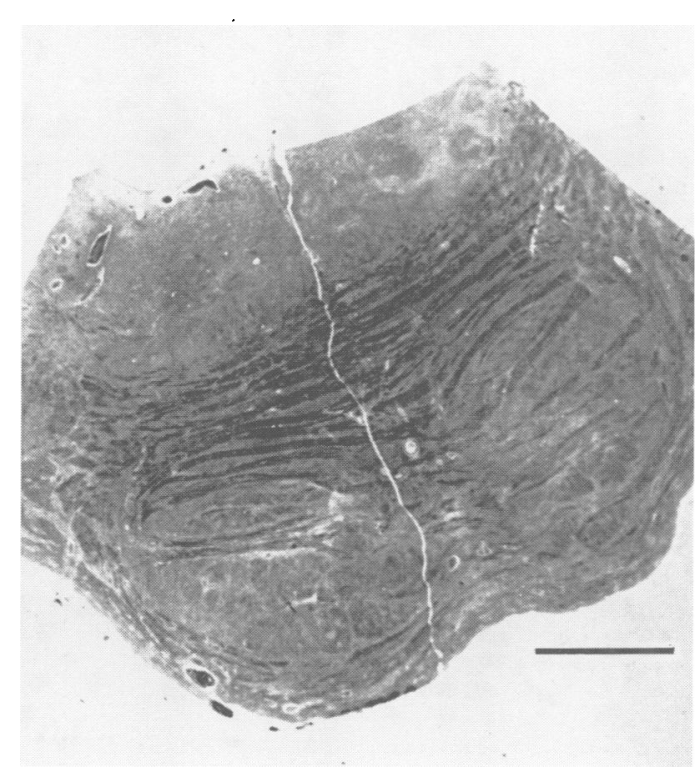

Figure 4 Luxol fast blue/haematoxylin-eosin staining showing demyelination of the ventral pons. Bar represents $4.8 \mathrm{~mm}$. 
Now the microscopic features (fig 5); a periodic acid Schiff stain demonstrated macrophages around blood vessels, inflammatory cells infiltrating the parenchyma, and some endothelial swelling. The neurons were spared. Immunohistochemistry with leucocyte common antigen, CD68, and glial fibrillary acid protein confirmed the presence of both lymphocytes and macrophages with glial reaction.

So the picture is of symmetric grey and white matter lesions (as on MRI), with spongy necrosis and demyelination, infiltration with macrophages and some lymphocytes, a degree of glial reaction, a tendency of vascular proliferation, and relative sparing of neurons.

\section{So what is the diagnosis?}

The clinical diagnosis at the time of the necropsy was of a meningoencephalomyelitis

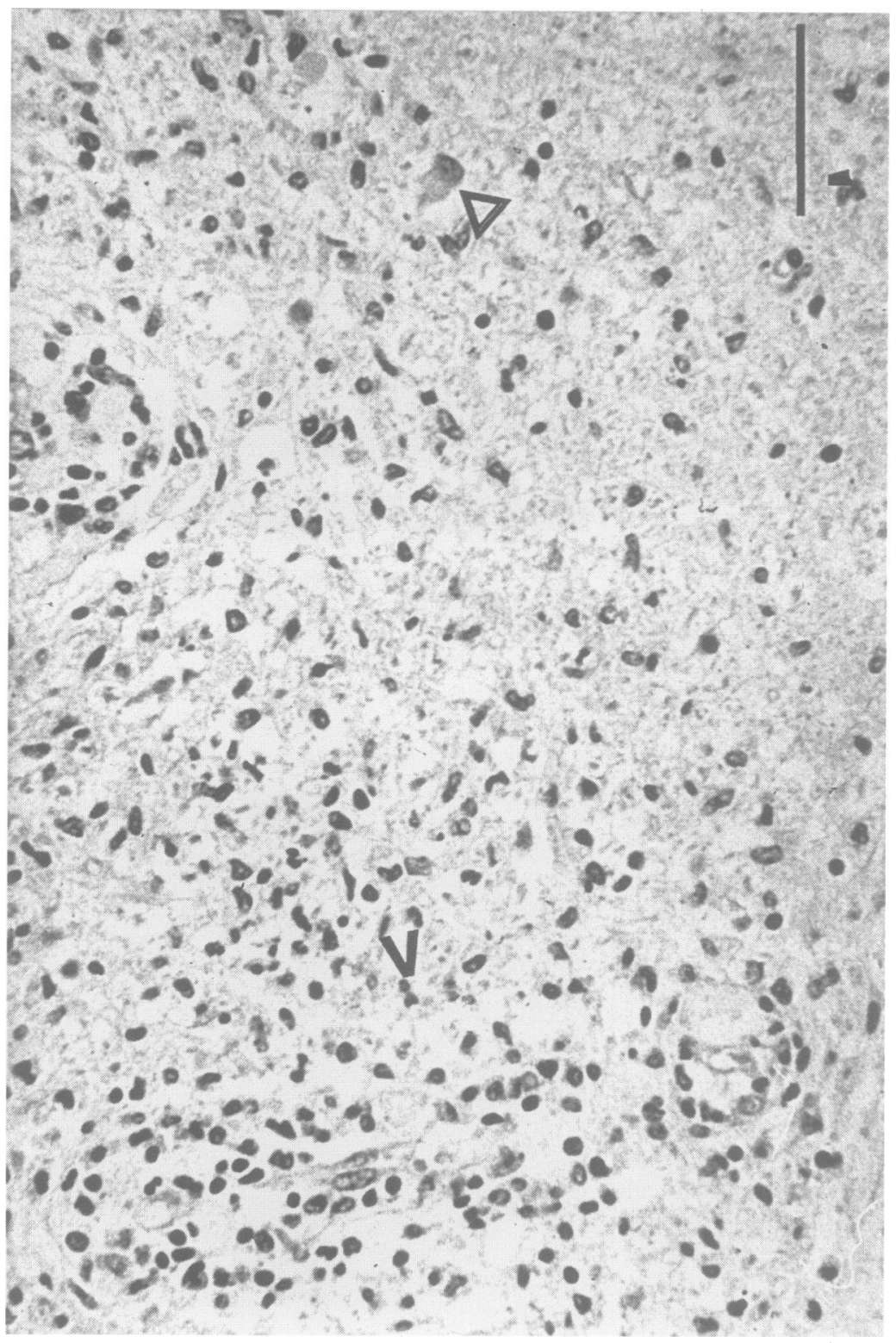

Figure 5 Periodic acid Schiff stain of the globus pallidus showing the histological appearance of the infiltrate in the parenchyma $(<)$ and sparing of a neuron $(\triangle)$. Bar represents $100 \mu \mathrm{m}$. caused by Mycoplasma pneumoniae. I did not find any characteristic petechiae or point haemorrhages, however, and, very importantly, there was no pneumonia. There was no Mycoplasma on microbiological investigation. So at the time of the necropsy no immediate diagnosis was apparent. The main clue was the degree of demyelination and the differential diagnoses included:

(a) Postinfectious encephalitis-There are normally two important features here; perivascular bleeding and inflammatory cells particularly plasma cells. Neither were found.

(b) Multiple sclerosis and all its variants-The lesions were clearly not plaques.

(c) Progressive multifocal leucoencephalopathyFor this diagnosis the demyelination should be associated with abnormal oligodendrocytes and bizarre astrocytes. In many cases Cowdry-like inclusions can be found and JC virus should be detectable. Neither the histological picture nor the virological studies we carried out supported this.

(d) Leucodystrophy-As an example adrenoleukodystrophy affects the cerebral hemispheric white matter much more diffusely, and affects the corpus callosum. The brain stem changes are normally only those of long tract degeneration. Gliosis is often denser than here.

(e) Pontine myelinolysis - The location of lesions outside the pons and the duration of the illness were not in harmony with this.

(f) Wernicke's encephalopathy-Our patient had many histological characteristics in common with this disease, but the absence of lesions in the mamillary bodies virtually excludes the diagnosis.

(g) Whipple's disease-In this disease macrophages are periodic acid Schiff positive not only around the vessels but also in the parenchyma and these were not found.

The histological features failed to exactly match any of this list, but closely fit the textbook description of Leigh's disease or subacute necrotising encephalomyelitis. ${ }^{8}$ The lesions were symmetric and showed striking vascular endothelial proliferation, astrocytosis, and proliferation of macrophages. The changes are similar to those of Wernicke's encephalopathy, but with sparing of the mamillary bodies. As already discussed by Professor Harrison, Leigh's disease is associated with mitochondrial abnormalities, ${ }^{9-11}$ but, regretfully, no muscle sample was taken. The pathological diagnosis was an adult form of Leigh's disease. ${ }^{12} 13$

We are grateful to Dr Cees Tigssen for preparing the details of the case.

1 Lindquist L, Linne T, Hansson LO, et al. Value of CSF analysis in differential diagnosis of meningitis: a study of 710 patients with suspected CNS infection. Eur 7 Clin Microbiol Infect Dis 1988:7;374-80.

2 Logigian EL, Kaplan RF, Steere AC. Chronic neurological manifestations of Lyme disease. New Engl ₹ Med 1990 323:1438-44.

3 Leigh DJ. Subacute necrotising encephalomyelopathy in an infant. $\mathcal{F}$ Neurol Neurosurg Psychiatry 1951;14:216-21.

4 Sipe JC. Leigh's syndrome. The adult form of subacute necrotising encephalomyelopathy with predilection for necrotising encephalomyelopathy with p
the brainstem. Neurology 1973;23:1030-8.

5 Menkes JH. The leukodystrophies. New Engl f Med 1990; 332:54-5. 
6 Kesselring J, Miller DH, Robb SA, et al. Acute disseminated encephalomyelitis. Brain 1990;113:291-302.

7 Kornips HM, Verhagen WIM, Prick MJ. Acute disseminated encephalomyelitis probably related to Clin Neurol Neurosurg 1993;95:59-63.

8 Adams JH, Duchen LW. Greenfield's neuropathology. 5th ed. London: Edward Arnold, 1992.

9 ed. London. Edward Ar Th, 1992 . Gilbert EF, Arya S, Chun R. Leigh's necrotizing encephalopathy with pyruvate carboxylase deficiency.

10 Coster R van, Lombes A, De Vivo DC, et al. Cytochrome c oxidate-associated Leigh syndrome: phenotypic features and pathogenetic speculations. F Neurol Sci 1991;104 97-111.

11 Sweeney MG, Hammans SR, Duchen LW, et al. Mitochondrial DNA mutation underlying Leigh's syndrome: clinical, pathological, biochemical and genetic studies of a patient presenting with progressive myostudies of a patient presenting with progre
clonic epilepsy. $\mathcal{F}$ Neurol Sci 1994;121:57-65.

12 Gray F, Louarn F, Gherardi R, et al. Adult form of Leigh's disease: a clinico-pathological case with CT scan examination. $₹$ Neurol Neurosurg Psychiatry 1984;47:121 1-15.

nation. F Neurol Neurosurg Psychiatry 1984;47:1211-15.
13 Khang-Loon Ho, Piligian JT, Chason JL. Adult form of subacute nicrotizing encephalomyelopathy. Arch Pathol Lab Med 1979;103:344-7. 